\title{
Metamaterials based on wedge-shaped electrodynamic structures
}

\author{
Vladimir Mitrokhin ${ }^{1}$, Georgy Kuvyrkin ${ }^{1}$, and Dmitry Ryzhenko ${ }^{1^{*}}$ \\ ${ }^{1}$ Bauman Moscow State Technical University, 105005 Moscow, Russia
}

\begin{abstract}
The paper studies a possibility of simulation of artificial composite media with negative values of the real part of the equivalent dielectric (magnetic) permittivity, by the use of segments of hollow composite waveguides with cylindrical guided waves in evanescent mode. Reactive evanescent fields of wedge-shaped waveguide eigenmodes are formed in the evanescent region before the critical section of the waveguide which separates the quasistatic field region from the distributing field of the evanescent waveguide mode. The possibility of simulation is determined by the equivalence of dispersion equation of the eigenmode propagation constant and the dispersion equation for the electric (magnetic) permittivity of plasma-like medium if cut-off frequency and electric (magnetic) plasma frequency of the medium are equal.
\end{abstract}

\section{Introduction}

In recent years, developers of microwave devices and antennas have been attracted by metamaterials and their use in microwave engineering [1-9]. Different combinations of infinitesimal electric and magnetic dipoles (wire structures, posts, bars, frames of various configurations, rings, etc.) are used for implementation of metamaterials. Often, waveguide sections operating in cutoff mode (evanescent mode) are used in combination with the above mentioned dipoles. Artificial composite medium proposed in [11] was made by placing a regular array of metallic posts between two parallel metallic plates. Further simplification of such model was achieved in [12] by using only one waveguide due to its dispersion properties.

\section{Metamaterials based on wedge-shaped electrodynamic structures}

In hollow metallic waveguides, $E_{m n}$ and $H_{m n}$ eigenmodes satisfy dispersion relation [10]:

$$
\Gamma=k \sqrt{\mu \varepsilon},
$$

where $\Gamma$ is eigenmode propagation constant; $k=\omega \sqrt{\varepsilon_{0} \mu_{0}}=2 \pi / \lambda$ - phase constant of empty space with $\varepsilon_{o}$ and $\mu_{o}$ parameters - dielectric permittivity and magnetic permeability of vacuum; $\omega$ - the angular frequency; $\lambda$ - the wavelength in vacuum; $\mu$ - the magnetic permeability (for hollow waveguide $\mu=1$ ); $\varepsilon_{\text {eff }}$ - the effective dielectric constant given by

$$
\varepsilon_{\text {eff }}=1-f_{\mathrm{cr}}^{2} / f^{2},
$$

where $f=\omega / 2 \pi$ is the operating frequency; $f_{c r}$ - the cutoff frequency of the considered type of eigenmode, e.g., $H_{m n}$.

Considering that the dispersion relation (1) with account of (2) is identical of that of a lossless plasma medium model with plasma frequency $f_{p e}=f_{c r}$ [10], a hollow metallic waveguide can be regarded as onedimensional plasma with respect to EM wave propagation along the axial direction of the waveguide. Moreover, the equivalence between waveguide field spread below cutoff frequency of the eigenmode and EM wave propagation in artificial plasma in the form of evanescent field is theoretically established in [13] and experimentally confirmed in [14]. The equivalence exists between waveguide and electric plasma for evanescent $H_{m n}$ modes of rectangular and circular waveguides. The discussed equivalence shows that medium with negative dielectric permittivity can be simulated by means of $H_{m n}$ modes at frequencies below cutoff, while medium with negative magnetic permeability can be simulated by means of $E_{m n}$ modes at frequencies below the corresponding cutoff.

With such approach double-negative medium is implemented by placing a periodic array of split ring resonators inside a rectangular or circular waveguides operating at frequencies below corresponding cutoff frequencies of $\mathrm{H}_{10}$ and $\mathrm{H}_{11}$ modes $[3,4,6]$. The periodic array of resonators is in fact artificial anisotropic magnetic plasma with permeability tensor [13]:

$$
\begin{gathered}
\vec{\mu}=\left(\begin{array}{ccc}
\mu_{\mathrm{pl}} & 0 & 0 \\
0 & 1 & 0 \\
0 & 0 & 1
\end{array}\right), \\
\mu_{\mathrm{pl}}=1-\frac{f_{\mathrm{pm}}^{2}-f_{0}^{2}}{f^{2}-f_{0}^{2}-i \gamma_{\mathrm{m}} f / 2 \pi},
\end{gathered}
$$

\footnotetext{
* Corresponding author: $\underline{\mathrm{dsr} @ \text {, bmstu.ru }}$
} 
where $f_{p m}$ is magnetic plasma frequency (when Lorenz model of the considered medium is used); $f_{o}$ is resonant frequency; $\gamma_{m}$ - magnetic plasma losses.

In such double-negative medium, the EM wave propagates with propagation constant

$$
\Gamma=k \sqrt{\left(1-f_{\mathrm{cr}}^{2} / f^{2}\right)\left(1-f_{\mathrm{pm}}^{2} / f^{2}\right)},
$$

In this structure, $H_{m n}$ modes can propagate at frequencies at which both brackets in the radical expression have the same sign. Backward wave is formed at frequencies lower than both $f_{c r}$ and $f_{p m}$. Forward waves propagate at all frequencies that are higher than both $f_{c r}$ and $f_{p m}$. Between the latter, there is a stopband. When $f_{c r}=f_{p m}$. all types of waves become propagating.

For $E_{m n}$ modes of the rectangular waveguide, dispersion relation can be expressed as

$$
\Gamma=k \sqrt{\varepsilon \mu_{\mathrm{eff}}},
$$

where $\mu_{\text {eff }}$ is effective relative magnetic permeability defined by

$$
\mu_{\mathrm{eff}}=1-f_{\mathrm{cr}}^{2} / f^{2}
$$

where $f_{c r}$ is the cutoff frequency of the considered $E_{m n}$ mode; for hollow waveguide, $\varepsilon=1$. Considering that the dispersion relation (3) with account of (4) is identical of that of a lossless plasma medium model with plasma frequency $f_{p m}=f_{c r}$ [14], a hollow metallic waveguide can be regarded as one-dimensional plasma with respect to EM wave propagation along the axial direction of the waveguide. In [14], the authors propose to simulate artificial medium with negative magnetic permeability by using a hollow square waveguide operating at frequencies below cutoff frequency of the lowest $E_{11}$ mode of the $E_{m n}$ eigenmode family.

Double-negative metamaterial medium is implemented by placing a periodic array of wires inside the square waveguide operating at frequencies below the cutoff frequency of the $E_{11}$ mode [13, 14]. The array structure is in fact artificial anisotropic magnetic plasma with dielectric permittivity tensor

$$
\begin{gathered}
\vec{\varepsilon}=\left(\begin{array}{ccc}
\varepsilon_{\mathrm{pl}} & 0 & 0 \\
0 & \varepsilon_{\mathrm{pl}} & 0 \\
0 & 0 & 1
\end{array}\right), \\
\varepsilon_{\mathrm{pl}}=1-\frac{f_{\mathrm{pe}}^{2}-f_{0}^{2}}{f^{2}-f_{0}^{2}-i \gamma_{\mathrm{e}} f / 2 \pi},
\end{gathered}
$$

where $f_{p e}$ is electric plasma frequency (when Lorenz model of the considered medium is used [6]); $f_{o}$ is resonant frequency; $\gamma_{\mathrm{e}}$ - electric plasma losses.

In such double-negative medium, the EM wave propagates with propagation constant

$$
\Gamma=k \sqrt{\left(1-f_{\mathrm{cr}}^{2} / f^{2}\right)\left(1-f_{\mathrm{pe}}^{2} / f^{2}\right)} .
$$

The $E_{m n}$ modes propagate at frequencies at which round parenthesis in the radical expression have the same sign. Backward wave is formed at frequencies lower than both $f_{c r}$ and $f_{p e}$. Forward wave propagates at frequencies that are higher than both of the above.

Regions with negative dielectric permittivity and magnetic permeability in nonhomogenious waveguides with spherical and cylindrical guided waves are implemented by means of evanescent reactive field of the corresponding eigenmodes before critical section of the waveguide separating the quasistatic field region from the distributing field of the evanescent waveguide mode $[1,10,19]$.

In wedge-shaped waveguides with cylindrical guided waves (in $\rho, \varphi, y$ coordinates) (Figure 1), Maxwell equations can be solved in the form of superposition of "electric' and "magnetic" waves, with their components determined via $\mathrm{U}$ and $\mathrm{V}$ potential functions [10]. Terms "electric" ( $\left\langle E_{m o} »\right)$ and "magnetic" $\left(« H_{m o} »\right)$ are quoted. It means that such splitting does not comply with the accepted waveguide classification, i.e. $y$ coordinate does not correspond to propagation direction. The waves are cylindrical and propagate in the direction of the $\rho^{0}$ radial coordinate. The $\mathrm{U}$ and $\mathrm{V}$ functions satisfy Helmholtz equations, with particular solutions given by

$$
\left.\begin{array}{l}
U \\
V
\end{array}\right\}=\left\{\begin{array}{l}
A \\
B
\end{array} Z_{P_{m}}(k \rho) \cos \left(p_{m} \varphi+\frac{m \pi}{2}\right)\right.
$$

where A and B are constant coefficients determined by excitation conditions; $Z_{m}(k \rho)$ is the radial dependence of the field satisfying boundedness condition at $k \rho \rightarrow 0$, radiation condition at $k \rho \rightarrow \infty$, and being continuous at the critical section [15]:

$$
k \rho=(k \rho)_{\mathrm{cr}}=\sqrt{p_{m}^{2}-0,25},
$$

where $p_{m}=m \pi /(2 \gamma)$ are eigenvalues determined from boundary conditions; $m$ - whole numbers; $2 \gamma$ - wedge angle.

Considering the above conditions, radial dependence should be written as:

$$
Z_{p_{m}}(k \rho)= \begin{cases}H_{p_{m}}^{(2)}\left[(k \rho)_{\mathrm{cr}}\right] J_{p_{m}}(k \rho), & k \rho \leq(k \rho)_{\mathrm{cr}}, \\ J_{p_{m}}\left[(k \rho)_{\mathrm{cr}}\right] H_{p_{m}}^{(2)}(k \rho), & k \rho \geq(k \rho)_{\mathrm{cr}},\end{cases}
$$

where $J p_{m}(k \rho)$ are cylindrical Bessel functions of the first kind and $p_{m}$ order, $\mathrm{Hp}_{m}{ }^{(2)}$ are cylindrical Hankel functions

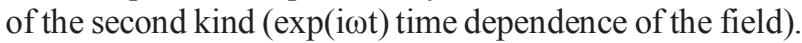

Transverse (relative to $\rho^{0}$ direction) $\mathbf{E}_{\perp}, \mathbf{H}_{\perp}$ field components constitute superposition of transverse EM field components for $\left\langle E_{m o}\right\rangle$ and $\left\langle H_{m o} »\right.$ waves:

$$
\begin{aligned}
& \mathbf{E}_{\perp}=\sum_{m} \mathbf{E}_{\perp \mathrm{e}}^{m}+\sum_{m} \mathbf{E}_{\perp m}^{m}, \\
& \mathbf{H}_{\perp}=\sum_{m} \mathbf{H}_{\perp \mathrm{e}}^{m}+\sum_{m} \mathbf{H}_{\perp m}^{m},
\end{aligned}
$$

where 


$$
\begin{gathered}
\mathbf{E}_{\perp \mathrm{e}}^{m}=\frac{1}{\rho} \frac{\partial^{2} U}{\partial y \partial \phi} \phi^{0}+\left(\frac{\partial^{2} U}{\partial y^{2}}+k^{2} U\right) y^{0}, \\
\mathbf{H}_{\perp \mathrm{e}}^{m}=-i \omega \varepsilon_{a} \frac{\partial U}{\partial \rho} \phi^{0}, \\
\mathbf{E}_{\perp m}^{m}=i \omega \mu_{a} \frac{\partial V}{\partial \rho} \phi^{0}, \\
\mathbf{H}_{\perp m}^{m}=\frac{1}{\rho} \frac{\partial^{2} V}{\partial y \partial \phi} \phi^{0}+\left(\frac{\partial^{2} V}{\partial y^{2}}+k^{2} V\right) \mathbf{y}^{0},
\end{gathered}
$$

Here, $\varphi^{0}, y^{0}$ are unit vectors along the $\varphi$ and $y$ coordinates.
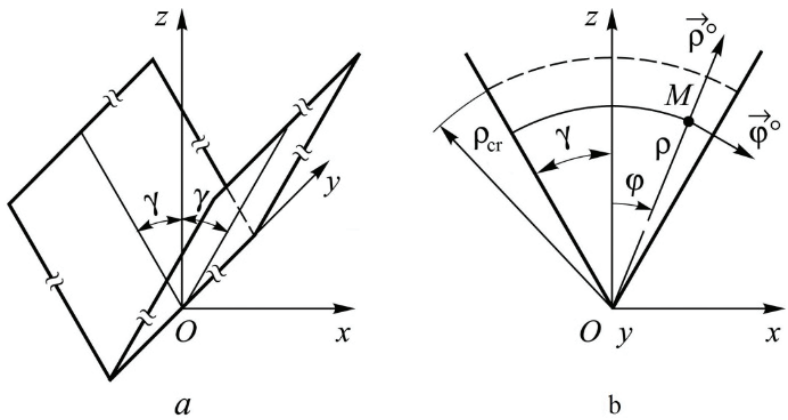

Fig. 1. Wedge-shaped waveguide: $a$ ) general view; b) in Cartesian and cylindrical coordinate systems.

The $\Gamma$ propagation "constant" of the eigenmodes is determined by means of logarithmic derivative of radial dependence [16]:

$$
\Gamma=-i k Z_{p_{m}}^{\prime}(k \rho) / Z_{p_{m}}(k \rho),
$$

where prime mark means full derivative of the argument. Since $\Gamma$ depends on $\rho$, The word "constant" is quoted.

Using asymptotic representation of Hankel function at $k \rho \rightarrow \infty$, it is possible to show that at narrow wedge angles

$$
\Gamma \approx k \sqrt{1-\left(p_{\mathrm{m}}^{2}-0,25\right)^{2} /(k \rho)^{2}}=k \sqrt{1-f_{\mathrm{cr}}^{2} / f^{2}},
$$

where

$$
f_{\mathrm{cr}}=c \sqrt{p_{\mathrm{m}}^{2}-0,25} /\left(2 \pi \rho_{\mathrm{cr}}\right),
$$

coinciding with (1) - (7) for regular waveguides. It allows to use the above methods for regular waveguides in this case too.

In the first example, let us consider a $\left\langle E_{10}\right\rangle$ mode, for which EM field components are given by:

$$
\begin{gathered}
E_{y}=k^{2} U=A k^{2} Z_{p_{1}}(k \rho) \sin \left(p_{1} \phi+\frac{\pi}{2}\right), \\
H_{\phi}=-i \omega \varepsilon_{a} \frac{\partial U}{\partial \rho}-A i \omega \varepsilon_{a} Z_{p_{1}}^{\prime}(k \rho) \sin \left(p_{1} \phi+\frac{\pi}{2}\right),
\end{gathered}
$$

Impedance for this type of wave is

$$
Z_{" E_{10} "}=E_{y} / H_{\phi}=Z_{0} k / \Gamma
$$

where

$$
Z_{0}=\sqrt{\mu_{a} / \varepsilon_{a}}
$$

$\mathrm{Z}_{0}$ is free space characteristic impedance with $\mu_{a}$ and $\varepsilon_{a}$ parameters.

Comparing (20) and (1) at $\mu=1$, we get

$$
-i Z_{p_{m}}^{\prime}(k \rho) / Z_{p_{m}}(k \rho)=\sqrt{\varepsilon_{\text {eff }}},
$$

From which

$$
\varepsilon_{\text {eff }}=-\left[Z_{p_{m}}^{\prime}(k \rho) / Z_{p_{m}}(k \rho)\right]^{2}
$$

If $k \rho \leq(k \rho)_{c r}$,

$$
\varepsilon_{\text {eff }}=-\left[J_{p_{m}}^{\prime}(k \rho) / J_{p_{m}}(k \rho)\right]^{2},
$$

If $k \rho \geq(k \rho)_{c r}$, expressions (7) and (1), given $\mu=1$, with account of relation (13) for propagation of the considered wave type after critical section, give

$$
-\frac{2}{\pi(k \rho)}\left[J_{p_{m}}^{2}(k \rho)+N_{p_{m}}^{2}(k \rho)\right]^{-1}=\sqrt{\varepsilon_{\text {eff }}},
$$

where $N p_{m}(\mathrm{k} \rho)$ are Neumann functions.

The relation gives for $k \rho \geq(k \rho)_{c r}$

$$
\varepsilon_{\text {eff }}=\frac{4}{\pi^{2}(k \rho)^{2}}\left[J_{p_{m}}^{2}(k \rho)+N_{p_{m}}^{2}(k \rho)\right]^{-2} .
$$

Consider the effective dielectric permittivity for $\left\langle E_{10}\right\rangle$ mode of the wedge-shaped waveguide with $2 \gamma=\pi / 2$ wedge angle. In this case,

$$
p_{m}=p_{1}=\pi /(\pi / 2)=2,
$$

then

$$
(k \rho)_{\mathrm{cr}}=\sqrt{p_{1}^{2}-0,25} \approx 2,
$$

Note that $(k \rho)$ variable in expression (28) and (30) can be expressed as:

$$
\mathcal{E}_{\text {eff }}= \begin{cases}-\left\{\left[\frac{f_{\mathrm{cr}}}{f}-J_{0}\left(\frac{2 f}{f_{\mathrm{cr}}}\right) / J_{1}\left(\frac{2 f}{f_{\mathrm{cr}}}\right)\right]^{-1}-\frac{f_{\mathrm{cr}}}{f}\right\}^{2}, & f<f_{\mathrm{cr}} \\ \left\{\left(\pi f / f_{\mathrm{cr}}\right)\left[J_{2}^{2}\left(2 f / f_{\mathrm{cr}}\right)+N_{2}^{2}\left(2 f / f_{\mathrm{cr}}\right)\right]\right\}^{-2}, & f>f_{\mathrm{cr}}\end{cases}
$$

The dependence is shown in the diagram in Figure 2. As we see, evanescent region of the wedge-shaped waveguide has negative dielectric permittivity. Doublenegative medium can be implemented by placing an array of split-ring resonators inside the wedge-shaped region according to the structure of the $\left\langle E_{10}\right\rangle(22) \mathrm{EM}$ field, i.e. resonator lattice plane should be perpendicular to the magnetic field vector lines.

In the second example, let us consider $\left\langle H_{10}\right\rangle$ mode, for which EM field components are given by: 


$$
\begin{gathered}
E_{\phi}=i \omega \mu_{a} \frac{\partial V}{\partial \rho}=B i \omega \mu_{a} k Z_{p_{1}}^{\prime}(k \rho) \cos \left(p_{1} \phi+\frac{\pi}{2}\right) \\
H_{y}=k^{2} V=B k^{2} Z_{p_{1}}(k \rho) \cos \left(p_{1} \phi+\frac{\pi}{2}\right)
\end{gathered}
$$

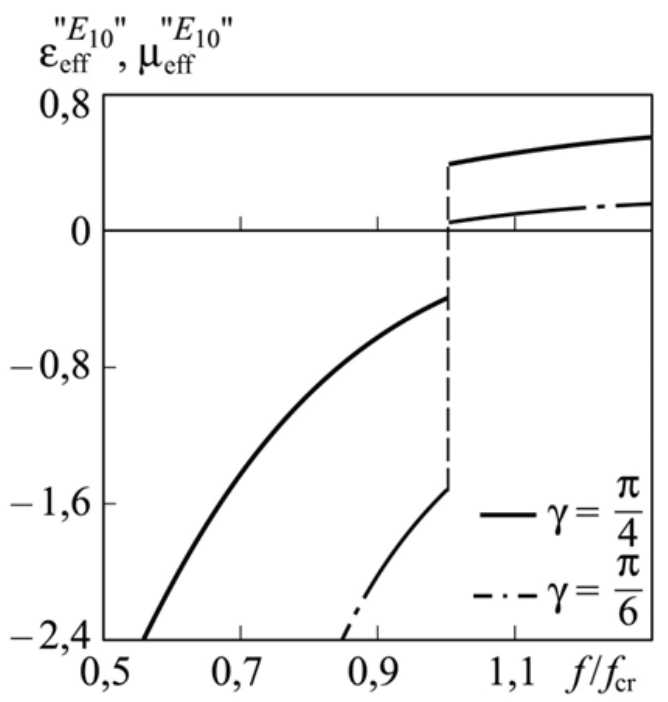

Fig. 2. Effective dielectric permittivity $\varepsilon_{\text {eff }} \bar{E}_{10} "$ and magnetic permeability $\mu_{\text {eff }} \mathrm{H}_{10} "$ of the wedge-shaped region at $\left\langle E_{10}\right\rangle$ and «H$H_{10} »$ waves, correspondingly, for different wedge angles.

Impedance for this type of wave is

$$
Z_{\text {" } H_{10} "}=E_{\phi} / H_{y}=-Z_{0} \Gamma / k,
$$

From expressions (6) and (19) at $\varepsilon=1$, we get

$$
-i Z_{p_{m}}^{\prime}(k \rho) / Z_{p_{m}}(k \rho)=\sqrt{\mu_{\mathrm{eff}}},
$$

From which

$$
\mu_{\mathrm{eff}}=-\left[Z_{p_{m}}^{\prime}(k \rho) / Z_{p_{m}}(k \rho)\right]^{2},
$$

For $k \rho \leq(k \rho)_{c r} \mu_{\text {eff }}$ is determined by formula (28), with $\varepsilon_{\text {eff }}$ substituted by $\mu_{\text {eff. }}$.

If $k \rho \geq(k \rho)_{c r}$, then, by comparing expressions (6) and (19), given $\varepsilon=1$ with account of relation (13) for propagation of the considered wave type after critical section, we get expression (30) for $\mu_{\text {eff }}$ if $\varepsilon_{\text {eff }}$ is substituted by $\mu_{e f f}$.

The effective dielectric permittivity for $\left\langle H_{10}\right\rangle$ mode of the wedge-shaped waveguide with $2 \gamma=\pi / 2$ wedge angle proves to be equal to (33) if $\varepsilon_{\text {eff }}$ is substituted by $\mu_{\text {eff }}$. This dependence is also shown in the diagram in Fig. 2. As we see, evanescent region of the wedge-shaped waveguide has negative magnetic permeability. Double-negative medium can be implemented by placing a periodic array of thin wires inside the wedge-shaped region according to the structure of the $\left\langle H_{10}\right\rangle$ (34) EM field, i.e. wires direction should coincide with the direction of the electric field.

\section{Conclusion}

The research that has been carried out allows to make conclusions as follows:

- a region with negative dielectric permittivity can be implemented based on hollow wedge-shaped waveguide at frequencies below the cutoff frequency of the $\left\langle E_{10}\right\rangle$ mode.

- a region with negative magnetic permeability can be implemented based on hollow wedge-shaped waveguide at frequencies below the cutoff frequency of the $\left\langle H_{10}\right\rangle$ mode.

The research work was supported by Ministry of Education and Science of the Russian Federation under state task No 8.4868.2017/8.9.

\section{References}

1. V.N. Mitrokhin, D.S. Ryzhenko and N.Yu. Fadeeva, Antennas 12(199), 4 (2013)

2. Y. Dong and T. Iston Microwave magazine 539 (2012)

3. D.S. Ryzhenko, V.N. Mitrokhin, V.A. Tyagunov, Microwave Bandpass Filter (Utility Model Patent RU 113077U, 2012)

4. D.S. Ryzhenko, V.N. Mitrokhin, V.A. Tyagunov, Microwave Bandstop Filter (Patent for Invention RF 2459321, 2012)

5. A.K. Sarychev and V.M. Shalaev, Electrodynamics of metamaterials (Moscow: Scientific world, 2011)

6. V.N. Mitrokhin, D.S. Ryzhenko and V.A. Tyagunov, Physics of Wave Processes and Radiotechnical Systems 14(3), 43 (2011)

7. V. Slyusar The First Line 3444 (2010)

8. V.N. Mitrokhin and D.S. Ryzhenko Vestnik SONIIR 4(26) 70 (2009)

9. Yu.V. Gulyaev, A.N. Lagar'kov and S.A. Nikitov, Herald of the Russian Academy of Sciences 78(5), 438 (2008)

10. N.S. Golubeva and V.N. Mitrokhin, Fundamentals of SHF Radio Electronics: Study Guide (Moscow: The Bauman University Publishing House, 2008)

11. D.R. Smith, W.J. Padilla, D.C. Vier, S.C. NematNasser and S. Schultz, Phys. Rev. Lett. 84(18), 4184 (2001)

12. R. Marques, J. Martel, F. Mesa and F. Medina, Phys. Rev. Lett. 89(18), 183901 (2002)

13. C. Camacho-Penalos, J. Esteban, T.M. MartinGinerero and E. Marques-Segyra, 27th ESA Antenna Technology Workshop (Santiago de Compostela, Spain, March 9-11) 461-468 (2004) 
14. J. Esteban, C. Camacho-Penalos, J.E. Page, T.M. Martin-Ginerero and E. Marques-Segyra, Transaction оп Microwave Theory and Techniques 53(4), 1506 (2005)

15. V.N. Mitrokhin, Radiotekhnika 4, 43 (1999)

16. V.N. Mitrokhin, Radiotekhnika 3, 62 (1985)

17. V.N. Mitrokhin, Radiotekhnika 7, 31 (1989)

18. V.N. Mitrokhin, Herald of the BMSTU. Instrument Engineering 1, 53 (1990)

19. V.N. Mitrokhin, Herald of the BMSTU. Instrument Engineering 1, 66 (1991)

20. V.N. Mitrokhin, Radiotekhnika 8, 64 (2002) 